\title{
Os cuidados paliativos na visão dos familiares de idosos residentes em uma instituição de longa permanência
}

\author{
Palliative care in the view of families of elderly residents in a long-term care institution
}

El cuidado paliativo a la vista de las familias de ancianos residentes en una institución de larga estancia

Mariana de Abreu Bombassaro ${ }^{1 *}$, Isabel Cristina Kirsten¹, Janaína Daiane Szemberg1, Márcio José de Almeida1.

\section{RESUMO}

Objetivo: Analisar qual é o entendimento dos familiares de idosos sobre os Cuidados Paliativos (CP) em uma Instituição de Longa Permanência para Idosos (ILPI). Métodos: Pesquisa exploratória, transversal e qualitativa. Informações coletadas por meio de entrevistas semiestruturadas e analisadas usando a técnica de Bardin. Seis entrevistados eram familiares de idosos em CP e oito eram familiares de idosos que não estavam em CP. Resultados: Entre os familiares de idosos que estão em CP, a maioria tem conhecimento sobre CP. Quando questionados acerca do entendimento dos CP, a maioria citou qualidade de vida (QV), cuidado multidisciplinar e identificou que o familiar está em CP. Sobre os cuidados que gostariam de receber, foram o tratamento não invasivo, alívio da dor, estar com a família e ter QV. Dentre familiares de idosos que não estavam em CP, foram esclarecidos termos relacionados à QV e diminuição de tratamentos invasivos. Quanto ao conhecimento, parte demonstrou conhecimento vago e outros não o tinham. Sobre o entendimento dos CP, abordou-se a QV. Conclusão: A maioria dos familiares possuem um bom entendimento dos CP com dificuldades na definição do conceito. Familiares dos idosos que estão em CP possuem entendimento melhor sobre o tipo de atenção recebida por eles.

Palavras-chave: Cuidados paliativos, Familiares, Idosos, Instituição de longa permanência para idosos.

\begin{abstract}
Objective: To analyze the understanding of family members of elderly people in a Long-Stay Institution (ILPI) about Palliative Care (PC). Methods: Exploratory, transversal and qualitative research in a ILPI in Curitiba. Data collection with semi-structured interviews. Analyzed with Bardin technique; 6 answered the interview for family members of elderly people in PC and 8 for family members of elderly people who are not in PC. Results: Among family members of elderly people who are in PC, most know about the topic. When asked about the understanding of $P C$, most cited quality of life (QL), multidisciplinary care and knew that the family member is in PC. About the care they would like to receive, was quoted non-invasive treatment, pain relief, being with family and having QL. Among family members of elderly people who are not in PC, were mentioned terms related to $Q L$ and reduction of invasive treatments. Part demonstrated vague knowledge and others had no knowing at all about PC. About the understanding of PC, QL was approached. Conclusion: Most family members have a good understanding of $\mathrm{PC}$, with difficulties in defining it. Family members of the elderly who are in $\mathrm{PC}$ have a better understanding of care.
\end{abstract}

Key words: Palliative care, Family, Elderly, Long-stay institution.

${ }^{1}$ Faculdades Pequeno Príncipe (FPP), Curitiba - PR. *E-mail: mari_bombassaro@hotmail.com 


\section{RESUMEN}

Objetivo: Analizar cuál es la comprensión de familiares de ancianos en una Institución de Larga Estancia para ancianos (ILPI) sobre Cuidados Paliativos (CP). Métodos: Investigación exploratoria, transversal y cualitativa en el ILPI de Curitiba. Recolección de datos con entrevistas semi estructuradas. Analizado con la técnica de Bardin. 6 respondieron la entrevista para familiares de ancianos en CP y 8 para familiares de ancianos que no están en CP. Resultados: Entre los familiares de ancianos que se encuentran en CP, la mayoría ha oído hablar de ella. Cuando se les preguntó sobre la comprensión de CP, la mayoría citó la calidad de vida (CV), la atención multidisciplinaria e identificó que el familiar está en CP. Sobre la atención que les gustaría recibir, eran tratamiento no invasivo, alivio del dolor, estar con la familia y tener CV. En cuanto al conocimiento, una parte demostró un conocimiento vago y otras no lo tenían. Sobre la comprensión de la CP, se abordó la CV. Conclusión: La mayoría de los familiares tienen un buen conocimiento del CP con dificultades para definirlo. Los familiares de los ancianos que se encuentran en CP tienen una mejor comprensión del cuidado.

Palabras clave: Cuidado paliativo, Familia, Adultos mayores, Institución de larga estancia para adultos mayores.

\section{INTRODUÇÃO}

A discussão em torno do tema Cuidados Paliativos é recente, mas seus primeiros registros datam da Idade Média, através dos Hospices, locais em sua maioria vinculados a igreja, que abrigavam indivíduos doentes ou qualquer pessoa que necessitasse de cuidados, tendo como objetivo principal o acolhimento e não necessariamente a cura (CASTILHO RK, et al., 2021).

O pontapé para o Movimento Hospice Moderno, mais próximo aos Cuidados Paliativos, como são conhecidos hoje, deu-se no final dos anos 1950 através da médica inglesa Cicely Saunders e um paciente vítima de um câncer terminal, em que a mesma se viu com a difícil tarefa de manejar corretamente a dor e os demais sintomas desse paciente. A partir disso, no ano de 1967, o St. Christopher's Hospice, além de oferecer assistência aos doentes, promoveu o desenvolvimento de pesquisas voltadas ao alívio da dor (RODRIGUES KM, 2018; CASTILHO RK, et al., 2021).

O primeiro conceito apresentado pela Organização Mundial de Saúde (OMS) para Cuidados Paliativos, em 1990, englobava apenas pacientes oncológicos e doentes incuráveis (CASTILHO RK, et al., 2021). Em 2002, o conceito foi revisado e ampliado para o que é conhecido hoje: os Cuidados Paliativos, cujo foco principal passou a ser a qualidade de vida em pacientes acometidos por doença ameaçadora a vida e também seus familiares, respeitando e priorizando os aspectos socioeconômicos e emocionais, bem como as crenças dos envolvidos (RODRIGUES KM, 2018).

A Academia Nacional de Cuidados Paliativos (ANCP) (2018), com o passar dos anos, vem influenciado a implementação do programa de Cuidados Paliativos pelos estados brasileiros. No entanto esse engajamento ainda é tímido, visto que tal cuidado ainda se concentra em hospitais. Além disso, o Brasil não possui uma política nacional de saúde que organize a construção dos Cuidados Paliativos. É grande a parcela dos profissionais da saúde que desconhecem técnicas de paliação e são escassas as publicações para esta área de atuação, e essa desinformação, por vezes se estende aos familiares dos pacientes. Essa informação se insere em um contexto em que o mundo vive uma intensa inversão da pirâmide etária e a população necessita cada vez mais desse tipo de cuidado (ANCP, 2018).

De acordo com dados divulgados pela Organização das Nações Unidas (ONU), atualmente o Brasil conta com 29,9 milhões de habitantes idosos, esse número representa $14 \%$ da população nacional. Espera-se um aumento, ainda mais expressivo, dessa população (com idade igual ou superior a 65 anos) para o ano de 2100 que passará a representar $40 \%$ do total de brasileiros. Esse envelhecimento populacional, atual e progressivo, exige atenção para o desenvolvimento de propostas de políticas públicas pertinentes (ALVES JED, 2019). Nesse cenário, composto por uma população que tende a reduzir a taxa de natalidade e progride 
no aumento da expectativa de vida, a principal consequência é o aumento da idade média e a prevalência de doenças crônico-degenerativas. Assim sendo, surge a necessidade de se pensar em custos assistenciais, visto que, o tratamento dessa população, por exemplo, com internações hospitalares e tecnologias, é cada vez mais oneroso (OLIVEIRA JAD, et al., 2020).

Junto ao aumento dos custos em saúde, outro fator a ser considerado com o aumento da idade média é a perda de autonomia e o aumento da dependência, que consiste na incapacidade de realizar atividades básicas e instrumentais da vida diária, necessitando assim de auxílio para a organização e gerência de suas vidas (CECCON RF, et al., 2021). O Brasil, segundo o IBGE, atualmente conta com 13,6\% da sua população idosa com algum grau de dependência em decorrência de doenças demenciais ou que comprometam habilidades de natureza motora, visual e auditiva, por exemplo (MENDONÇA JBM, et al., 2021).

A família é compreendida como principal responsável pelo cuidado do idoso, junto com a sociedade e o Estado, conforme dispositivos legais vigentes na Constituição Federal de 1988, na Política Nacional do Idoso de 1994 e no Estatuto do Idoso de 2003 (ASSOCIAÇÃO NACIONAL DOS MEMBROS DO MINISTÉRIO PÚBLICO DE DEFESA DOS DIREITOS DAS PESSOAS COM DEFICIÊNCIA E IDOSO, 2015). No entanto, questões como mudanças na estrutura familiar, como a diminuição do número de filhos e inserção da mulher no mercado de trabalho, a vulnerabilidade familiar, a sobrecarga do familiar cuidador e a exigência de cuidados contínuos que levam o cuidador a abdicar de suas próprias necessidades demonstram a imprescindibilidade de outros pontos de apoio (NUNES DP, et al., 2019; NASCIMENTO HG e FIGUEIREDO AEB, 2019).

Neste cenário surgem as Clínicas de Retaguarda e as Instituições de Longa Permanência para Idosos (ILPIs) que atuam na prestação de serviços de cuidados pós-agudos, com foco em pacientes que necessitam de uma rede de assistência para continuidade dos cuidados. A proposta do modelo refere-se aos períodos de convalescença e recuperação, otimizando a capacidade funcional do paciente, preparando-o para cuidados de longo prazo e minimizando desfechos inapropriados, que resultam nas reinternações hospitalares (POLISAITIS AEG, 2018).

O termo ILPI foi proposto pela Sociedade Brasileira de Geriatria e Gerontologia (SBGG) no ano de 2003, para substituir o termo "asilo". O nome é uma adaptação do termo Long-Term Care Institution utilizado pela OMS (POLISAITIS AEG, 2018). Nas ILPIs é indispensável oferecer uma assistência gerontogeriátrica voltada para as necessidades dos seus residentes. Para isso, é necessária uma equipe multiprofissional (médicos, psicólogos, fisioterapeutas, terapeutas ocupacionais) com o intuito de averiguar o estado funcional, mental e social do idoso. Assim, é possível traçar um plano de cuidados com ações de prevenção de doenças, ações curativas, paliativas e de reabilitação (PATROCINIO WP, 2016).

Nesse contexto, o objetivo principal do estudo é analisar qual é o entendimento dos familiares a respeito dos Cuidados Paliativos em uma Instituição de Longa Permanência para Idosos.

\section{MÉTODOS}

Este estudo consiste em uma pesquisa de natureza exploratória, transversal e de abordagem qualitativa. O estudo foi conduzido em uma ILPI particular que possui 27 apartamentos, sendo 11 duplos e 16 individuais. O tempo médio de permanência dos idosos residentes na ILPI é de cinco anos. No dia 30/04/2020 abrigava 38 idosos. Foi selecionado um familiar por idoso residente durante o período definido de coleta, totalizando 38 convidados a participar da entrevista. Haja vista o possível constrangimento sobre o tema por parte dos familiares foi acionada a coordenação da ILPI para realizar o primeiro contato. Disso, obtiveram 15 respostas positivas à participação da entrevista, com uma posterior desistência, totalizando 14 familiares de idosos entrevistados. Destes, 6 tinham o seu familiar idoso em CP e 8 não o tinham em CP.

A coleta de informações foi realizada por meio de duas entrevistas semiestruturadas em meio digital, cujo primeiro roteiro foi direcionado a familiares de idosos que não estão em Cuidados Paliativos e o segundo roteiro aos familiares de idosos que estão em Cuidados Paliativos. A coleta das informações sobre quais idosos residentes que estavam e não estavam em Cuidados Paliativos foi feita diretamente com a equipe da ILPI. Para a análise das informações, utilizou-se a técnica de Bardin. 
Os participantes assinaram o Termo de Consentimento Livre e Esclarecido (TCLE) e estavam cientes dos riscos e benefícios da entrevista. Todas as ações foram embasadas e regidas pela Resolução 466/12 do Conselho Nacional de Saúde (CSN) e o Projeto de Pesquisa foi submetido no Comitê de Ética e Pesquisa (CEP), que o aprovou sob o parecer número: 4.278.974.

\section{RESULTADOS E DISCUSSÃO}

\section{Conhecimento dos Familiares de Idosos que estão em Cuidados Paliativas}

Entre os que responderam à entrevista direcionada aos familiares de idosos que estão em Cuidados Paliativos, cinco eram do sexo feminino e um era do sexo masculino, idades entre 32 e 62, a maioria eram filhos, autônomos e com ensino superior completo. Os motivos pelos quais os familiares optaram pela ILPI são: problemas com cuidadores em casa (falta de cuidado, má relação com a cuidadora), uso abusivo de álcool pelo idoso, esgotamento emocional e cuidado melhor na ILPI. O tempo de residência dos idosos na ILPI é entre seis meses e três anos. A frequência das visitas possui uma média de 1 a 3 vezes por semana, com relativa redução com a pandemia. As doenças dos idosos, citadas pelos familiares, foram: câncer renal, diabetes mellitus, cirrose alcoólica, encefalopatia aguda, síndrome Parkinsoniana, pressão baixa, infarto agudo do miocárdio, depressão, doença de Alzheimer e um dos idosos não possui comorbidades.

Em vista do questionamento se o participante já ouviu falar sobre os Cuidados Paliativos, a maioria respondeu afirmativo, conhecimento resultante da comunicação efetiva entre família e os profissionais. Furtado MEM e Leite DMC (2017) afirmam que essa comunicação é uma qualidade inerente à equipe. Uma comunicação falha, mesmo com o objetivo de proteção do ente querido, pode resultar em sofrimento na unidade familiar. Apenas um participante afirmou desconhecer o tema; porém, no decorrer da entrevista foi evidenciado que este soube identificar o que eram os Cuidados Paliativos, apenas não relacionou o termo ao significado. Os autores afirmam que muitos, mesmo compreendendo o prognóstico desfavorável do familiar, ainda não conseguem definir o que seriam os Cuidados Paliativos.

Quando questionados acerca do entendimento dos Cuidados Paliativos, a maioria citou a melhoria na qualidade de vida que está englobada na definição dos Cuidados Paliativos pela OMS, como cita Castilho RK, et al. (2021) além do cuidado multidisciplinar como aspecto fundamental. Houve também uma resistência inicial em relação aos Cuidados Paliativos, o que lembra a reflexão de Gawande A (2015); segundo ele, para a maioria das pessoas a morte só vem após uma longa batalha contra a enfermidade e todos lutam com a incerteza de quando a morte chegará: como e quando aceitar que a incessante batalha está perdida.

Todos os participantes identificaram que o familiar está em Cuidados Paliativos, aqui fica clara a importância da percepção do paciente sobre a doença o que facilita a compreensão do quadro e auxilia no processo de tratamento, Cain CL, et al. (2018) afirmam que discordâncias na comunicação do processo de tratamento podem resultar em grande impacto nas prioridades dos pacientes e familiares, tanto nas preocupações e necessidades como em outras dimensões do cuidado. Para esses entrevistados o cuidar não se limita ao diagnóstico e tratamento, sendo necessário explorar dentro do contexto em que esse indivíduo insere suas expectativas, bem como as de seus entes queridos, de modo a integrar os fenômenos fisiológicos e emocionais nas decisões.

O impacto na vida relatado pelos familiares resumiu-se no idoso estando bem e feliz, com serenidade no olhar e com um tratamento menos invasivo, todas essas características culminam na melhoria da qualidade de vida do idoso. Roth AR e Canedo AR (2019) confirmam que o objetivo é priorizar o alívio dos sintomas e sofrimento diante de condições ameaçadoras à vida, o que envolve o bem-estar emocional, espiritual, físico, mental e psicológico, além do planejamento do cuidado, de acordo com Dy SM, et al. (2017). Os Cuidados Paliativos surgem para frear essa necessidade de cura absoluta, mudando o objetivo da incessante luta contra a doença e a visão da morte como uma batalha perdida, como afirma Machado RS, et al. (2016), para um cuidado mais humano visando melhoria no bem-estar físico, mental, social e espiritual.

Todos os entrevistados demonstraram interesse em Cuidados Paliativos em caso de indicação médica, resultado que não coincide com a literatura científica, o que pode ter sido influenciado por: 1) durante o roteiro da entrevista as autoras explicaram ao familiar o conceito do termo Cuidados Paliativos, o que pode ter gerado 
a homogeneidade das respostas e 2) o estudo foi realizado em uma instituição privada bem aceita pelos familiares. Ao serem indagados acerca de quais cuidados gostariam de receber em caso de doença ameaçadora à vida, os principais foram o tratamento não invasivo, alívio da dor, estar com a família e ter qualidade de vida. Características que coincidem com os Cuidados Paliativos.

Sobre a curiosidade a respeito do tema obtiveram-se quatro respostas positivas, em razão do aumento da expectativa de vida refletindo em um maior interesse nos assuntos relacionados ao "envelhecer com qualidade", como citado por Gomes ALZ e Othero MB (2016). E duas respostas negativas visto que um entrevistado já possuía um conhecimento que considerava suficiente e o outro não possuía nenhum questionamento no momento da entrevista.

\section{Conhecimento dos Familiares de Idosos que não estão em Cuidados Paliativas}

O grupo dos familiares de idosos que não estão em Cuidados Paliativos foi composto por oito entrevistados, sendo seis do sexo feminino e dois do sexo masculino, com idades entre 33 e 64 anos. A maioria são filhos dos idosos e casados, empregados com carteira assinada ou autônomos. Com ensino superior completo e pós graduação, um possui mestrado. $O$ tempo de residência na ILPI varia de seis meses e três anos e meio. A frequência das visitas varia de uma vez por semana a todos os dias e houve redução com a pandemia.

Os motivos pelos quais optaram pela permanência do idoso na ILPI foram a doença de Alzheimer, custo alto de cuidadores, depressão e tentativa de suicídio, filhos que não moram no Brasil, evolução da demência fronto-temporal, melhoria da qualidade de vida, dinâmica familiar afetada pela presença de cuidadores em casa, evolução da doença de Parkinson. As doenças dos idosos citadas pelos familiares foram hipertensão, fibrilação atrial, enfisema pulmonar, hérnia de disco, depressão, doença de Alzheimer, cardiomegalia, câncer intestinal, arritmia, anemia, demência, demência fronto-temporal, diabetes, doença de Pick, doença de Parkinson.

Acerca do conhecimento sobre os Cuidados Paliativos, três conheciam o tema e citavam termos importantes relacionados aos conceitos de qualidade de vida e diminuição de tratamentos invasivos. Três demonstravam um conhecimento vago sobre o tema e dois não tinham conhecimento do termo.

Um estudo sistemático da literatura revisou o conhecimento, consciências e percepções dos Cuidados Paliativos da população em geral entre os anos de 2003 e 2019, nos países da Nova Zelândia, Irlanda, Reino Unido, Coreia e Suécia. A conclusão foi que existe pouca consciência e diversas percepções equivocadas sobre o tema e essas descobertas permanecem constantes ao longo do tempo, a despeito do crescimento dos Cuidados Paliativos (PATEL P e LYONS L, 2020). Assim, ao comparar com o presente trabalho, apesar das respostas positivas, a maioria ainda desconhece ou não compreende totalmente o termo, mesmo no universo de entrevistados com alta escolaridade.

Quando questionados acerca do entendimento a respeito do tema foram obtidas respostas como não prolongar uma situação que não terá melhora e ofertar qualidade de vida, abordadas por três entrevistados, evidenciando um bom entendimento sobre os Cuidados Paliativos. Outras respostas como manter as coisas como elas estão e que nos Cuidados Paliativos também tem sofrimento foram obtidas em entrevistas específicas. Três entrevistados manifestaram interesse em saber o que é, evidenciando apoiar o assunto. Como mostrado nas falas abaixo; por questões de ética os entrevistados foram citados como: E2, E3.

"Cuidados Paliativos significam que você mantém cuidados nesse paciente/pessoa dentro de uma normalidade que ela vinha sendo tratada, mas não toma nenhuma atitude invasiva no sentido de prolongar uma vida que não está tendo qualidade de vida" (E2).

"Você diminuir a medicação e oferecer outro tipo de qualidade de vida, um cuidado diferente do que seria com medicamentos muito fortes" (E3).

Diante dessas entrevistas, evidencia-se uma compreensão que o modelo hospitalocêntrico vigente hoje, com a incessante busca pela cura e na negação da morte, como afirmam Gomes ALZ e Othero MB (2016) 
nem sempre é a melhor alternativa para uma efetiva melhoria no quadro clínico do paciente. O panorama começa a se alterar para uma preferência pelo cuidado ao fanatismo pelo tratamento (FURTADO MEM e LEITE DMC, 2017).

Ao serem questionados sobre a existência dos Cuidados Paliativos na ILPI, a maioria das repostas foram afirmativas. Tal fato demonstra uma boa comunicação da equipe junto à família, que fica a par do cuidado ofertado ao seu familiar. O Conselho Regional de Medicina do Estado de São Paulo destaca a "excelente comunicação" entre a equipe profissional, o paciente e os familiares como fundamental para o enfrentamento da doença. O Conselho, aponta ainda que, em Cuidados Paliativos a ênfase deve ser atribuída na qualidade das relações, sendo imprescindível a comunicação honesta e completa (FURTADO MEM e LEITE DMC, 2017). O desconhecimento do cuidado foi contemplado por um único participante mas, em segunda análise, reparou-se uma incongruência em relação a sua autopecepção sobre o cuidado.

Em relação ao interesse em receber Cuidados Paliativos futuramente as respostas unanimes foram positivas. Nesse momento da entrevista já havia sido explanado, por parte das entrevistadoras, os conceitos, princípios e benefícios básicos dos Cuidados Paliativos, pois a disseminação dos conhecimentos acerca dos Cuidados Paliativos também era um objetivo da pesquisa. Aqui, fica evidente que existe uma compreensão da crescente necessidade dos Cuidados Paliativos em decorrência do aumento da expectativa de vida e o anseio por envelhecer com qualidade de vida, com atenção e cuidado em caso de doença ameaçadora à vida.

Ao abordar a descrição dos cuidados que o entrevistado gostaria de receber, caso enfrentasse uma doença ameaçadora à vida, foram obtidas respostas muito alinhadas com os conceitos dos Cuidados Paliativos. Por exemplo, cuidado humanizado, aferido pela resposta:

$$
\text { "olha, basicamente receber um cuidado humanizado" (E2). }
$$

Apenas o cuidado, como cita Franco HCP, et al. (2017) é um fator que leva a humanização e, consequentemente, a qualidade de vida, termo que esteve presente na resposta da maior parte dos entrevistados, constituindo um fator muito importante na escolha de abordagem de tratamento. Outras respostas como alívio da dor e estar com a família também foram contempladas, demonstrando a importância desses pontos.

Ao indagar se os entrevistados possuíam curiosidade sobre os Cuidados Paliativos em idosos as respostas obtidas foram positivas e negativas. Apenas em quatro entrevistas confirmou-se interesse ou curiosidade acerca do assunto.

\section{CONCLUSÃO}

A partir desse trabalho foi possível concluir que, em sua maioria, os familiares de idosos que residem em uma ILPI possuem um bom entendimento sobre os Cuidados Paliativos. Em relação àqueles que desconhecem o termo, existe um interesse e abertura em relação a conhecê-lo e também um apreço e uma concordância em relação aos princípios dos Cuidados Paliativos. Muitos dos entrevistados reconhecem a importância da equipe multidisciplinar e afirmam que gostariam de receber um cuidado focado no alívio do sofrimento e da dor, na melhor qualidade de vida, menos tratamentos invasivos e o interesse de estar perto dos seus familiares. Como já era esperado, os familiares dos idosos que estão em Cuidados Paliativos, possuem um entendimento maior sobre a atenção prestada e quais os serviços fornecidos. Em contrapartida, dentre os familiares de idosos que não estão em Cuidados Paliativos, houve um número maior de negativas ao questionamento sobre o conhecimento do tema.

\section{AGRADECIMENTOS}

Nosso apreço à Dra. Claudia Cibele Ranzi de Oliveira e à Me. Luciane Maria Kallinowski Rocha de Miranda que foram sempre solícitas em relação às nossas dúvidas, disponibilizaram tempo para participarem de reuniões e abriram as portas da ILPI. Agradecemos a Professora Elaine Rossi, exemplo de profissional dedicada que nos acompanhou do início ao fim indicando ajustes e literaturas científicas valiosas. À ILPI e aos familiares participantes, nosso muito obrigada, por toda atenção e disponibilidade. 


\section{REFERÊNCIAS}

1. ALVES JED. Envelhecimento populacional no Brasil e no mundo: novas projeções da ONU. Revista Longeviver, 2019; 3: 5-9.

2. ASSOCIAÇÃO NACIONAL DOS MEMBROS DO MINISTÉRIO PÚBLICO DE DEFESA DOS DIREITOS DAS PESSOAS COM DEFICIÊNCIA E IDOSO (AMPID). I Seminário Internacional sobre Políticas de Cuidados de Longa Duração para Pessoas Idosas. 2015. Disponível em: http://www.ampid.org.br/v1/i-seminario-internacional-sobrepoliticas-de-cuidados-de-longa-duracao-para-pessoas-idosas/. Acesso em: 20 jan. 2020.

3. ACADEMIA NACIONAL DE CUIDADOS PALIATIVOS (ANCP). Panorama dos Cuidados Paliativos no Brasil. 2018. Disponível em: https://paliativo.org.br/wp-content/uploads/2018/10/Panorama-dos-Cuidados-Paliativos-no-Brasil2018.pdf. Acesso em: 18 jan. 2019.

4. CAIN CL, et al. Culture and Palliative Care: Preferences, Communication, Meaning, and Mutual Decision Making. J Pain Symptom Manage, 2018; 55(5): 1408-1419.

5. CASTILHO RK, et al. Manual de cuidados paliativos da Academia Nacional de Cuidados Paliativos (ANCP). 3. ed. Rio de Janeiro: Atheneu, 2021.

6. CECCON RF, et al. Envelhecimento e dependência no Brasil: características sociodemográficas e assistenciais de idosos e cuidadores. Ciência \& Saúde Coletiva, 2021; 26(1):17-26.

7. DY SM, et al. Palliative Care for Cancer Survivors. Med Clin North Am., 2017; 101(6):1181-1196.

8. FRANCO HCP, et al. Papel da enfermagem na equipe de cuidados paliativos: a humanização no processo da morte e morrer. Revista Gestão e Saúde, 2017; 12(2): 48-61.

9. FURTADO MEM, LEITE DMC. Palliative care seen from the point of view of the lung cancer patient's families. Interface, 2017; 21(63): 969-80.

10. GAWANDE A. Mortais: nós, a medicina e o que importa no final. 1ed. Rio de Janeiro: Objetiva, 2015; 259p.

11. GOMES ALZ, OTHERO MB. Cuidados Paliativos. Instituto de Estudos Avançados da Universidade de São Paulo, 2016; 30(88).

12. MACHADO RS, et al. Finitude e morte na sociedade ocidental: uma reflexão com foco nos profissionais de saúde. Cultura de los Cuidados, 2016; 20(45).

13. MENDONÇA JMB, et al. O sentido do envelhecer para o idoso dependente. Ciência \& Saúde Coletiva, 2021; 26(1):5765.

14. NASCIMENTO HG, FIGUEIREDO AEB. Demência, familiares cuidadores e serviços de saúde: o cuidado de si e do outro. Ciência \& Saúde Coletiva, 2019; 24(4): 1381-1392.

15. NUNES DP, et al. Cuidadores de idosos e tensão excessiva associada ao cuidado: evidências do Estudo SABE. Revista Brasileira de Epidemiologia, 2019; 21Suppl 02: e180020.

16. OLIVEIRA JAD, et al. Longevidade e custo da assistência: o desafio de um plano de saúde de autogestão. Revista Ciencia \& saúde coletiva, 2020; 25(10): 4045-4054.

17. PATEL P, LYONS L. Examining The Knowledge, Awareness, And Perceptions Of Palliative Care In The General Public Over Time: A Scoping Literature Review. Am J Hosp Palliat Care, 2020; 37(6): 481-487.

18. PATROCINIO WP. O cuidado de idosos em Instituições de Longa Permanência e em Centros-Dia. Kairós Gerontologia, 2016; 18(19): 77-97.

19. POLISAITIS AEG. Cuidados pós agudos: como estão inseridas nas políticas de saúde brasileira as Instituições de Longa Permanência para Idosos (ILPIs) e as clínicas de retaguarda?. Dissertação (Mestrado em Gestão para Competitividade) - Gestão da Saúde. Escola de Administração de Empresas de São Paulo da Fundação Getulio Vargas, São Paulo, 2018; (1): 43.

20. ROTH AR, CANEDO AR. Introduction to Hospice and Palliative Care. Prim Care, 2019; 46(3): 287-302.

21. RODRIGUES KM. Princípios dos cuidados paliativos - Porto Alegre: SAGAH, 2018. 